\title{
1 Evolution of Selective RNA Processing and Stabilization operons in cellulosome-harboring Clostridium spp.
}

3 Yogendra Bhaskar ${ }^{1,3, *}$, Mohammadhadi Heidari B., ${ }^{1,3}$, Chenggang $\mathrm{Xu}^{2}$, Jian $\mathrm{Xu}^{1,3, *}$

4

$5 \quad{ }^{1}$ Single-Cell Center and CAS Key Laboratory of Biofuels and Shandong Key Laboratory of

6 Energy Genetics, Qingdao Institute of Bioenergy and Bioprocess Technology, Chinese

7 Academy of Sciences, Qingdao, Shandong, 266101, China

$8 \quad{ }^{2}$ College of Life Science, Shanxi University, Taiyuan, Shanxi, 030006, China

$9{ }^{3}$ University of Chinese Academy of Sciences, Beijing, 100049, China

11 "Correspondence: Tel: +86 5328066 2651; Fax: +86 53280662654

12 E-mail address: 2014in-yogendra@qibebt.ac.cn and xujian@qibebt.ac.cn

Key words: stem-loop structure; stoichiometry of protein complexes; cellulosome; operon 


\section{Abstract}

17 In selective RNA processing and stabilization (SRPS) operons, the stoichiometry of 18 encoded proteins is determined by their respective 3'-end stem-loops (SLs), yet the evolution 19 of this mechanism remains elusive. In cellulosomal operons of Clostridium spp., we show 20 that the SLs and their associated genes form a monogamy companionship during the operon 21 evolution. Based on $\Delta \mathrm{G}$ of such SLs, we propose CoSLOE (Composite SL-based Operon Evolution) model with evolutionary ratio (ER) $>1$ or $<1$ for positive or negative selection of SRPS operons. In the composite SL- $\Delta \mathrm{G}$-based tree (CoSL-tree) of cellulosomal operons, when traversing from leafs to the root nodes, ERs reveal diversifying/positive selection towards a less efficient cellulosomal system, consistent with glycoside-hydrolase gene variation both in-operon and genome-wide. A similar pattern is followed by the ATPase operon and the majority of orthologous SRPS operons genome-wide, suggesting conservation among operons in such selection. Thus SRPS operons via their transcript-stabilizing noncoding elements are highlighting a link between operon stoichiometry and operon evolution. 


\section{Introduction}

In bacterial genomes, $\sim 50 \%$ of the genes are organized and regulated in the form of operon (Osbourn \& Field et al., 2009). Within an operon, to ensure proper absolute and relative abundance of the component genes, one strategy adopted by certain bacteria is selective RNA processing and stabilization (SRPS), where the RNA molecule is cleaved by ribonuclease into fragments, and then with the involvement of the specific cis-elements (Stem-loops), mature mRNA transcripts stabilize to differential gene expression and eventually to the protein complex (Rochat et al., 2013). The SRPS mechanism controls operons that encode a variety of key protein complexes and regulatory pathways such as the glycolysis pathway, maltose transport system, cellulosome complex and photosynthetic apparatus (Newbury et al., 1987, Klug et al., 1993, Ludwig et al., 2001, Xu et al., 2015).

Using the cellulosome-encoding cip-cel operon of Clostridum cellulolyticum (Ccel) as a model, we showed that the stem-loops generally located at the 3'-end of regulated genes precisely regulate structure and relative abundance of the subunit-encoding transcripts processed from a primary polycistronic RNA (Xu et al., 2015). Importantly, the "ratio" of subunit-encoding transcripts for the cip-cel operon, which quantitatively specifies cellulosome stoichiometry, appears to be encoded by the genome (i.e., organism-specific) and insensitive to alteration of culture conditions, since change among glucose, cellobiose and cellulose did not result in ratio change (Xu et al., 2015). These findings revealed a key role of such stem-loops (SLs; i.e., all such SLs present in a SRPS operon) in specifying proper function of SRPS-operon-encoded protein complexes (or metabolic pathways). Moreover, they strongly suggest potential links between the structure and function of these stem loops to organismal evolution.

However, key questions remain unanswered: ( $i$ ) how do these SLs evolve? How conserved are these SLs among orthologous operons? What is the nature of such conservation? (ii) What is the link in evolution between these SLs and their companion genes in SRPS operons? (iii) What roles do these SLs play in the evolution of SRPS operons? Are these roles conserved for SPRS operons at a genome-wide scale? How similar or divergent are these roles across different genomes? Can evolution of SRPS operons be quantitatively modeled via these SLs? Here in cellulosomal operons of Clostridium spp., based on $\Delta \mathrm{G}$ of such SLs, we propose CoSLOE (Composite SL-based Operon Evolution) with evolutionary ratio (ER) $>1$ or $<1$ for positive or negative selection of SRPS operons. In the CoSL-tree of cellulosomal operons, when traversing from leafs to the root nodes, ERs reveal diversifying/positive selection towards a less efficient cellulosomal system, consistent with glycoside-hydrolase gene variation both in-operon and genome-wide. A similar pattern is followed by the ATPase operon and the majority of orthologous SRPS operons genome-wide, suggesting conservation among operons in such selection. Thus SRPS operons via their transcript-stabilizing non-coding elements are highlighting a link between operon stoichiometry and operon evolution.

\section{Materials and Methods}




\subsection{Prediction of the stable stem-loops}

SLs in C. cellulolyticum were predicted via the following steps (Bhaskar et al., 2021). (i) Prediction of motifs using RNAmotif (Macke et al., 2001); (ii) Estimation of free-energy and RNA secondary structure using RNAfold (Hofacker, 2003); (iii) Genome mapping of the predicted SLs; (iv) Screening of the SLs based on their stability for highly stable SLs. These stable SLs were then mapped to the operon map of the respective species, followed by functional classification based on the derived classification rules, whereby the SRPS operons were identified. Genome-encoded ratios were predicted for these SRPS operons using the $\Delta \mathrm{G}$ of the harbored SLs (Bhaskar et al., 2021).

\subsection{Calculation of the $\Delta$ G-based ratio for an SRPS operon}

Ratios were calculated in the SRPS operon using the $\Delta \mathrm{G}$ (free-energy) of the SLs present in and flanking the operon (Fig. 1A). For example, the ratio for a four-gene operon (with SLs found after first two genes and at the end of operon) "Gene-1 $(\Delta \mathrm{G} 1)$ : Gene-2 $(\Delta \mathrm{G} 2)$ : Gene3 : Gene-4 ( $\Delta \mathrm{G} 4)$ " would be " $\Delta \mathrm{G} 1: \Delta \mathrm{G} 2: \Delta \mathrm{G} 4: \Delta \mathrm{G} 4$ ". To normalize the ratio, $\Delta \mathrm{G}$ of all SLs in an operon were divided by the sum of all $\Delta \mathrm{G}$ (Table S1).

\subsection{Phylogenetic analysis of the cellulosomal and the ATP synthase operons}

The genomes and associated annotations of 13 cellulosome operon-harboring Clostridial species including Ruminiclostridium cellulolyticum H10 (Ccel; NC_011898.1), Ruminiclostridium papyrosolvens DSM 2782 (Cpap; GCF_000175795.2), Clostridium saccharoperbutylacetonicum (Csac; NC_020291.1), Clostridium sp. BNL1100 (Cbnl; GCF_000244875.1), Clostridium felsineum DSM 794 (Cfel; GCF_002006355.1), Ruminiclostridium josui JCM 17888 (Cjos; GCF_000526495.1), Ruminiclostridium cellobioparum subsp. termitidis CT1112 (Cter; GCF_000350485.1), Clostridium acetobutylicum ATCC 824 (Cace; NC_015687.1), Clostridium cellulovorans 743B (Cloc; NC_014393.1), Ruminiclostridium hungatei DSM 14427 (Chun; GCF_002051585.1), Clostridium puniceum DSM 2619 (Cpun; GCF_002006345.1), Clostridium roseum DSM 7320 (Cros; GCF_002006215.1) and Ruminiclostridium cellobioparum DSM 1351=ATCC 15832 (Ccell; GCF_000621505.1) (Table S1), were downloaded from NCBI. Cellulosome operons from Cpap, Csac, Cbnl, Cfel, Cjos, Cter, Cace, Cloc, Chun, Cpun, Cros and Ccell were identified by the available annotation and BLAST (Altschul et al., 1990), where the cipcel operon (encoding the cellulosome) from $\mathrm{Ccel}$ was used as a query with the e-value cutoff of 1e-5. Organismal phylogeny (16S-tree) of these species was derived using the 16S rRNA sequence, where all positions containing gaps and missing data were eliminated, which resulted in a total of 1,326 positions in the final multiple-sequence alignment. Phylogenetic analyses for the cellulosome and the ATPase operons were conducted in MEGA7 (Kumar et al., 2016) via the Maximum Likelihood method.

The $\Delta$ G-based dendrogram of SLs was performed using the pvclust (Suzuki \& Shimodaira, 2006) package in R (CRAN http://cran.r-project.org/) (Fig. S1A). To calculate the SLs' $\Delta \mathrm{G}$-based dendrogram (CoSL-tree), $\Delta \mathrm{G}$ of all SLs in an operon were divided by the sum of all $\Delta \mathrm{G}$, which generated a normalized proportion for an operon, and empty cells (i.e., 
values are "non-applicable") were replaced by the average value of that proportion while clustering (Table S1). The normalized $\Delta \mathrm{G}$ proportions of 13 clostridia species were supplied to pvclust with the Euclidean distance method and the ward.D2 hierarchical clustering, with bootstrapping for 1000 times. The $\mathrm{Ka} / \mathrm{Ks}$ values for genes were calculated using the Codeml tool of PAML package (Yang et al., 2007).

\subsection{Structural alignment analysis of orthologous SLs}

The orthologous SLs from the cellulosomal operons and the ATP synthase operons were aligned structurally and sequence-wise using the LocARNA alignment and folding tool (Smith et al., 2010, Will et al., 2012). Evolution of the orthologous SLs was shown using the multiple-alignment of SL sequences and dot-bracket notations.

\subsection{Derivation of Composite Stem-Loop-based Operon Evolution (CoSLOE) model}

The CoSLOE model was described via an equation that calculates the evolutionary ratio (ER):

$$
\frac{G 1}{G 2} \times \frac{S 1}{S 2} \times \frac{C V 1}{C V 2}=E R
$$

where G1 and G2, S1 and S2, CV1 and CV2 are the number of genes, number of SLs and coefficient of variations (CV) respectively, in the two operons. CV is the ratio of standard deviation (RatiosD) and mean ( $\overline{\text { Ratio }})$ of the $\Delta \mathrm{G}$-based ratio of an operon.

\section{$3 \quad$ Results}

\subsection{Phylogenetic analysis of the SLs in cellulosome-encoding SRPS operons from 13 Clostridial genomes}

To probe the role of SLs in the function and evolution of SRPS operons at the wholegenome scale, we developed an approach to identify the SRPS operons based on the genomewide predicted stable stem-loops (SLs) and then use the free-energy $(\Delta \mathrm{G})$ of these stable SLs to calculate ratios of SRPS operons (Bhaskar et al., 2021) (Fig. 1A). The $\Delta$ G-based ratios were calculated for the cellulosome complex operon (cip-cel) in Ccel, which can model stoichiometry of the encoded complex. To probe how this mechanism has evolved, we extended the analysis to twelve additional mesophilic Clostridial spp.: C. papyrosolvens (Cpap), C. saccharoperbutylacetonicum (Csac), C. sp. BNL1100 (Cbnl), C. felsineum (Cfel), C. josui (Cjos), C. termitidis (Cter), C. acetobutylicum (Cace), C. cellulovorans (Cloc), C. hungatei (Chun), C. puniceum (Cpun), C. roseum (Cros), and C. cellobioparum (Ccell; Table S1). These operons are orthologous, as indicated by orthology of genes, functional conservation of encoded proteins and the global similarity in operon structure. Our $\Delta \mathrm{G}$-based method predicted 7, 7, 5, 5, 5, 6, 5, 4, 3, 3, 3 and 3 SLs in Cbnl, Cpap, Cjos, Cter, Ccell, Chun, Cloc, Cace, Cpun, Cros, and Cfel respectively (Table S1). The $\Delta$ G-based ratio for these Clostridial species were also highly skewed, e.g., the ratios of Cbnl and Cpap are "24.4:-26.3:-25.9:-25.9:-25.9:-15.3:-15.3:-21.2:-21.2:-18.3:-21.5:-21.5” and “-23.6:-26.3: - 
25.3:-25.3:-25.3:-16.7:-16.7:-16.8:-16.8:-23.5:-23.9:-23.9” respectively. Similarly, Ccell and Cter exhibit identical ratios, so do Cace, Cfel and Cros (Fig. 1B; Table S1).

To probe how such operon properties have evolved, the $\Delta \mathrm{G}$-based proportions of all harbored SLs in an operon (which we termed "composite SLs" or CoSL) were used to generate a dendrogram (CoSL-tree; Fig. S1A). CoSL-tree was then compared to the $16 \mathrm{~S}$ rRNA-based tree (16S-tree; i.e., the organismal phylogeny; Fig. S1B). Predicted ratios from the 13 cellulosomal operons were combined to form a data matrix, which was then used for the hierarchical clustering with 1000 iterations to generate the ratio-based tree (Methods). Intriguingly, the species were classified differently in the two clades derived from CoSL-tree (Fig. S1A) and 16S-tree (Fig. S1B). For example, (i) Cace and Cros are in Clade 1 of CoSLtree, yet found in Clade 2 of 16S-tree; (ii) Cpun is an out-group in CoSL-tree, whilst Cfel is an out-group in 16S-tree; (iii) Cros and $\mathrm{Cfel}$ are clustered in CoSL-tree yet distantly apart in 16S-tree. Such difference between CoSL-tree and 16S-tree indicates the deviation of SRPS operon evolution from organismal taxonomy.

\subsection{Gene-SL relationship during evolution of Clostridial cellulosomal operons}

To probe the roles of SLs in cellulosomal operon evolution, seven orthologous SLs were first identified in the intergenic regions of the 13 orthologous cellulosomal operons, via comparison of their sequences, structures and organization in the operons (Fig. 1C; Fig. 2; Fig. S2). However, not all the Clostridial species harbor similar numbers of orthologous SLs and at identical positions (Fig. 1C; Table S1): 7 SLs in Cbnl and Cpap, 6 in Ccel and Chun, 5 in Cjos, Cter, Ccell and Cloc, 4 in Cpun and Cace and 3 in Cfel, Cros and Csac. The presence of these SLs suggests SPRS mechanisms in these 13 cellulosomal operons (for Cloc, the role of multiple promoters is also involved (Doi et al., 1998)).

Interestingly, although the region between a SL and its associated genes can be inserted by another gene, the SLs are always positioned with their associated genes in a sequential fashion that is conserved among a set of orthologous operons. Thus, to probe the gene-SL relationship, orthologous SLs were aligned via sequence and structural similarity (Fig. S2). Compatible base pairs (in the stem sequences) were found in SL-1, 2, 3, 5 and 7, underscoring the structural similarity among the orthologous SLs (Fig. 2). Specifically, $(i)$ SL-1 is present in all the Clostridial species (except Cloc), and SL-1 and 2, in their respective clades, are of similar length and identical $\Delta \mathrm{G}$ to other orthologous SLs, yet show higher variation in their loop sequences (Fig. 2A, B; Fig. S2A, B); (ii) SL-3 shows less sequence variation in the two clades than SL-1 and 2, possibly due to its role as terminator SLs (Fig. 2C; Fig. S2C); (iii) SL-4, 5 and 6 are clade-specific, as they are absent in Clade 2 except the SL-4 in Cloc (Fig. 2D; Fig. S2D, E, F); (iv) similar to SL-3, SL-7 carries a low level of sequence variation (Fig. 2E; Fig. S2G). Such variation in SL sequence and structure depicts their evolutionary distance.

Intriguingly, a dockerin-encoding gene, located at the $7^{\text {th }}$ position of operon in Cloc, the $9^{\text {th }}$ in subclade of Ccel-Cjos-Cbnl-Cpap (except Cjos) and the $12^{\text {th }}$ in Subclade 1.1 species (except Chun) is always controlled by the orthologous SL-5 (Fig. 1C, 2D; Fig. S2E). Similarly, a cellulase-encoding gene, situated at distinct positions among cellulosomal 
operons, is controlled by an orthologous SL-3. In addition, clade-specific derivative homologous SLs in the cellulosomal operons also show such loyalty with their respective companion genes, e.g., (i) Cloc harbors an extra cellulase-encoding gene carrying SL-3A (homologous to SL-3; Fig. S2H); (ii) SL-2A is found in Ccel and Chun, similar to SL-2 (Fig. S2I); (iii) SL-7A is found in Csac, which is similar to SL-7 and works as terminator to the operon (Fig. S2J). These observations suggest monogamy as one feature of the gene-SL relationship during evolution of SRPS operons.

\subsection{The Composite Stem-Loop based Operon Evolution (CoSLOE) model for SPRS operons}

Taking advantage of the link between SLs and evolution of operon, we propose Composite Stem-Loop based Operon Evolution (CoSLOE) for the SRPS operons (Fig. 3). The model consists of (Equation I): $(i)$ the number of genes in the operon $(\mathrm{G})$, where the addition of one gene shows the positive selection, while an equal number of genes suggests neutral operons; (ii) the number of SLs (S), which plays crucial roles in regulation, stabilization and termination of genes; (iii) variance of $\Delta \mathrm{G}$ of the SLs in operons (CV), where multiple SLs with distinct free-energy together specify and control the stoichiometry of gene expression. Therefore, the evolutionary ratio (ER) of an operon with respect to the other operons is,

$$
\frac{G 1}{G 2} \times \frac{S 1}{S 2} \times \frac{C V 1}{C V 2}=E R(\text { for ideal condition, } \mathrm{ER}=1)
$$

where G1 and G2, S1 and S2, CV1 and CV2 are the number of genes, number of SLs and coefficient of variations (CV) respectively, in the two operons. CV is ratio of standard deviation and mean of the ratio of $\Delta G$ of SLs for an operon. Positive or purifying selection of the operon is indicated by ER $>1$ and ER $<1$ respectively, while ER of 1 corresponds to neutral selection (i.e., ideal condition).

\subsection{CoSLOE reveals selection pressure on the cellulosomal operons}

To probe their evolution, pairwise ERs for the 13 cellulosomal operons in CoSL-tree were derived via CoSLOE (Equation I; Fig. 3). In Subclade 1.2 (Fig. 1C), (i) Cbnl and Cpap show ER of 1.01 and 0.99 with each other respectively, suggesting that the selection pressure is almost neutral and $\mathrm{Cbnl}$ is positively selected towards the root; (ii) the next nearest species is Cjos, which lacks one gene and two SLs possibly due to the deletion or horizontal transfer of genes, shows the ER of 0.57, 0.57 and 0.52 with Cpap, Cbnl and Ccel respectively (Table 1A), i.e. equally separated from all the three clostridia; (iii) however, the $\mathrm{Ka} / \mathrm{Ks}$ values, at the gene level selection, for the first gene of Cjos are 1.42, 1.45, and 1.5 with Ccel, Cbnl, Cpap respectively (Table S2), suggesting the first gene of these operons is under positive selection towards Cjos; (iv) the operon ER for Ccel is 1.931 .10 and 1.10 with Cjos, Cbnl and Cpap respectively (Table 1A), which depict the positive selection with the addition of a new gene at $11^{\text {th }}$ place in operon (Fig. S5). Taken together, in Subclade 1.2 of CoSL-tree, species are under positive selection while going from Cpap to Ccel (Fig. 1C), and 
also while going from Cros to Cace (due to the much higher Cace-Cros ER of 7.76 than CfelCros ER of 1.05; Table 1B).

In Subclade 1.1, the SLs in Ccell operon are more similar to Cter than to Chun, i.e., the operon ERs for Cter-Ccell and Chun-Ccell are 1.10 and 1.83 respectively (Table 1A), while those for Cter-Chun and Ccell-Chun are 0.60 and 0.55 respectively. Thus Ccell and Cter are in purifying selection, while Subclade 1.1 is under positive selection towards Chun (similar to as Subclade 1.2; Fig. 1C; Fig. S5).

The Clade 2 species in CoSL-tree are more dynamic in evolution than Clade 1, in that they show more variable number of genes and SLs. Cloc, Csac and the out-grouped Cpun exhibit a certain degree of similarity to the Clade 1 species, but feature the addition of new SLs such as SL-3A and SL-7A (homologous to SL3 and SL7 respectively; Fig. 1C). Moreover, their cellulosomal operons are distinct, e.g., Cpun and Csac operons harbor no cohesin, glycoside hydrolase $(\mathrm{GH})$ or dockerin genes. In fact, ERs for Cpun and Csac versus Cloc are 1.37 and 2.11 respectively (Table 1B), consistent with positive selection.

Notably, if the ERs are calculated without considering SLs (and the CV) in Equation I, then the number of genes by itself is not sufficient to detect the selection. For example, in Subclade 1.2 (Cpap-Cbnl-Cjos-Ccel; Cros-Cfel-Cace), the equal number of genes would suggest an ER of 1, which however is misleading. Therefore, in computing CoSL-based ER, the SLs are essential for deriving ERs in CoSLOE.

\subsection{The CoSLOE model of cellulosomal operons is supported by variation in enzyme genes}

In CoSLOE, purifying/negative selection occurs when the tree is traversed from the root to the leaf nodes, and diversifying/positive selection takes place when traversing from leafs to the root nodes (Fig. 4). In the cellulosomal operon (Fig. 1C), positive evolution takes place in the Ccel-Cjos-Cbnl-Cpap direction (root to leaf), in the Chun-Cter-Ccell direction and in the Cace-Cros-Cfel direction respectively, with the root node being the most positively selected and the leaf nodes the most negatively selected.

To probe the biological significance of these findings, the genome-wide numbers of carbohydrate-active enzymes (CAZymes) and carbohydrate-binding module (CBM) were compared, since these enzymes are major parts of the cellulosomal system(Busch et al., 2017). For example, in Subclade 1.2, for the Ccel, Cjos, Cbnl and Cpap genome (which exhibit $>95 \%$ similarity in 16S rRNA sequences; Fig. 1C, 6A), $(i)$ the CAZymes (including glycoside hydrolases or GHs, carbohydrate esterases or CEs, and polysaccharide lyases or PLs) harbored is 111 (94 GHs, 13 CEs, 4 PLs), 116 (92 GHs, 19 CEs, 5 PLs), 127 (103 GHs, 19 CEs, 5 PLs) and 122 (103 GHs, 16 CEs, 3 PLs) respectively (Dassa et al., 2017), exhibiting an overall pattern of increase; (ii) for GH5 (Ccel: 7; Cjos: 7; Cbnl: 8; Cpap: 7), GH9 $(13,14,14,14)$ and $\operatorname{GH} 43(9,13,13,13)$, an increase in number is apparent when traversing from root to the leave nodes (Ccel-Cjos-Cbnl-Cpap); (iii) a similar pattern (i.e., increase in number) is observed in $\operatorname{CBMs}(54,59,67,71)$ and to a less degree, dockerins $(69$, 72, 88, 68) (Dassa et al., 2017) (Fig. 4A). Thus Ccel is an outlier in terms of the genome- 
wide CAZyme number. Moreover, of $26 \mathrm{~Kb}$ in size, the cellulosomal operon of Ccel is the largest (Fig. S5; Cjos: 22.5Kb; Cbnl: 25Kb; Cpap: 25Kb), and harbors unique genes such as pectin degrading enzymes (Pagès et al., 2003, McDonald et al., 2008) (Rgl11Y) and longer hybrid linkers (Pinheiro et al., 2008) that join cohesins to scafoldins. Similarly, in the CaceCfel-Cros cluster, the Cace cellulosomal operon harbors one additional enzyme (Sialidase; Fig. 4B) yet lacks cellulosomal complex activity (Sabathé et al., 2002), in opposite to Cros and $C f e l$ which are used for the retting process (Angelini et al., 2013). These observations are consistent with CoSLOE-derived positive selection of the Ccel cellulosomal operon.

Similarly, in Subclade 1.1, the cellulosomal operon of Chun uniquely harbors a

\subsection{Evolution of the ATP synthase operons via CoSLOE is similar to the cellulosomal operons}

CoSL-tree of the ATP synthase operons is similar to that of the cellulosome operons (Fig. 5A; Fig. 1C), except that Cpun is clustered with Chun in the former. Notably, within each of the Chun-Cell-Cter, Ccel-Cpap-Cbnl-Cjos and Cros-Cace-Cfel subclades, operon sequences are nearly $100 \%$ similar, and the gene sequences of subunit alpha and beta are conserved across 13 species (Fig. 5A). The less variation in gene sequences (than cellulosome operon) among 13 Clostridium species is probably due to the strict functional conservation of the ATP synthase complex.

As in cellulosome operons, gene-SL relationship was probed in the ATPase operons. Three orthologous SLs were predicted in ATPase operon, where (i) SL-1 is preserved in Chun, Cjos, Cbnl, Cpap and Ccel (always flanking at 3' UTR of subunit C); (ii) SL-2 is present at 3' UTR of subunit alpha in Cpun, Cjos, Cbnl, Cpap, Ccel, and Csac; (iii) SL-3 is conserved throughout the 13 species at the 3' UTR of epsilon chain and terminating the operon; (iv) Only one SL is predicted in Cace, Cfel, Cros, and Cloc, suggesting that their ATP synthase operons seem not regulated by the SRPS mechanism (Fig. 5B). Taken together, these associations between genes and SLs show their relationship, which is consistent with the observation in cellulosome operons.

As for ER, in Clade 1, the ERs for Cter-Cpun, Ccell-Cpun and Chun-Cpun are 0.34, 0.41 and 0.80 respectively (Table S3), revealing negative selection towards leaf nodes in Clade 1 (Fig. 5A), i.e. Ccell and Cter appear to undergo purifying selection, while Chun is positively selected towards Cpun (ER for Cpun-Chun: 1.24; Table S3). In Subclade 2.1, similarly, ERs of Cjos-Ccel, Cbnl-Ccel and Cpap-Ccel are 0.94, 0.93, 1.20, respectively (Table S3), i.e. the overall flow of Cjos-Cbnl-Ccel is consistent with positive selection, except for Cpap (Fig. 5A).

Interestingly, the ATP synthase operons exhibit an evolution pattern similar to the cellulosome operons, by positive selection in the Cter-Ccell-Chun and Cjos-Cbnl-Cpap-Ccel, direction (purifying selection in the reverse direction; Fig. 5A). Since ATP synthase operon is 
functionally conserved in most species (Neupane et al., 2019), less variability was present in the genes and SLs. However, the root species of Subclade 1.1 (Chun), 1.2 (Ccel) and 2.1 $(\mathrm{Cloc})$ harbors smaller operons, longer operons and an additional enzyme at the 3' UTR region, respectively (Fig. 5A), consistent with the evolutionary pattern suggested by the observations in cellulosome operons.

\subsection{Genome-wide application of COSLOE reveals the direction of organismal selection}

The evolutionary flow in a tree represents the different directions that species follow due to the selection-pressure on them, during evolution. To probe SL-driven evolutionary selection-pressure, orthologous SRPS operons were probed using CoSLOE. However, due to the lack of orthology among SRPS operons in 13 species, operon evolution was probed cladewise in CoSL-tree (Fig. 1C), i.e. Subclade 1.2 (Ccel-Cjos-Cbnl-Cpap). In Subclade 1.2, orthologous SRPS operons are scattered across the genomes of Ccel, Cjos, Cbnl and Cpap which are in the form of chromosome (Cbnl and Ccel) or contigs (Cpap-31 and Cjos-2; Fig. 6A; Table S4).

Here, five out of the 25 SRPS operons, i.e., Op617, Op622, Op716, Op863 and Op1745, follow the Ccel-Cjos-Cbnl-Cpap direction (black arrows; Fig. 6A). Interestingly, the other 80\% SRPS operons show the positive selection flow in the Cpap-Cbnl-Cjos-Ccel direction, i.e. from the leaf nodes to the root nodes, which is similar to the cellulosomal cluster evolutionary flow, e.g., for Op142, Op376 and Op898 (red arrows; Fig. 6A). These observations suggest that the SRPS mechanism, although evolutionarily conserved, can reveal selection-pressure that is distinct from organismal phylogeny.

\section{Discussion and conclusion}

In existing frameworks of operon evolution, coding sequences (i.e., subunits of protein complex or components of metabolic pathway encoded by the operon) have been thought to play a major role. They can drive structural variation and functional adaptation of operons towards a specific niche (Gogarten et al., 2002, Francino et al., 2012), for example, by deletion or insertion of the whole genes or via synonymous/non-synonymous mutations of their sequences. However, it remains elusive whether non-coding elements play a role in such adaptation of operons.

The stoichiometry of SRPS operons, found genome-wide, can be modeled based on the genome sequence of SLs alone (Bhaskar et al., 2021), suggesting a quantitative model of evolution at the whole operon level, in parallel to the evolution at the individual coding gene level (e.g., Ka/Ks (Kimura et al., 1968)). Based on $\Delta \mathrm{G}$ of such SLs, we proposed CoSLOE, with evolutionary ratio (ER) $>1$ or $<1$ for positive or negative selection of SRPS operons. In the CoSL-tree of cellulosomal operons, when traversing from leafs to the root nodes, ERs reveal diversifying/positive selection towards a less efficient cellulosomal system, consistent with glycoside-hydrolase gene variation both in-operon and genome-wide. A consistent pattern is followed by the ATPase operon and the majority of orthologous SRPS operons genome-wide, suggesting conservation among operons in such selection. Therefore, CoSLOE provides a new layer of insights into operon evolution that is distinct from existing models 
(Fig. 6B). Specifically, (i) Driving forces: for individual genes, mutation, recombination, genetic drift and selection are known evolution drivers; for SRPS operons, in addition to addition/deletion/mutation of genes, CoSLOE introduces SLs a previously unrecognized driver. (ii) Theoretical models: in addition to the known models of gene evolution (neutral theory (Nei et al., 2005)) and operon evolution (selfish operon theory (Lawrence \& Roth et al., 1996), co-regulation model (Price et al., 2005) and piece-wise model (Fani et al., 2005)), CoSLOE provides a new framework for quantitatively modeling SRPS operon evolution. (iii) Rate of selection: CoSLOE compares rate of selection between two orthologous operons, which is conceptually similar to $\mathrm{Ka} / \mathrm{Ks}$ or $\mathrm{dN} / \mathrm{dS}$ which compares between orthologous genes. (iv) Outcome of selection: gene evolution generally results in changed protein sequence, yet the operon evolution depicted by CoSLOE results in altered ingredient or stoichiometry of the whole protein complex or metabolic pathway. ( $v$ ) Direction of selection: just like $\mathrm{Ka} / \mathrm{Ks}$ for orthologous genes, CoSLOE offers strategy to model the direction for orthologous operons. (vi) Phylogeny: a tree based on $\triangle \mathrm{G}$ of SLs of SRPS operons can model the selection of operon and organism, in contrast to 16S-rRNA gene trees that model organismal phylogeny. (vii) Underlying sequence: instead of relying on coding sequences, CoSLOE takes advantage of the non-coding sequences to model operon evolution, and highlights the role of cis-elements in shaping operon evolution and organism adaptation. (viii) Origin of operons: CoSLOE suggests the SLs (and their relationship with associated genes) as a key player in the original formation of operon structure, in addition to horizontal gene transfer, deletion of intervening genes and addition of ORFan genes (Price et al., 2006).

Notably, we have tested CoSLOE on just 13 Clostridial species, and expansion of the model to a broader range of species is limited by the paucity of experimental data and lack of computational approaches to identify SPRS operons. Therefore, to what degree the model is applicable across microorganisms is not yet clear, and answer to this question is perhaps ultimately dependent on the breath and boundary of SPRS mechanism. Despite these limitations, for SRPS operons, our findings here reveal the link between operon stoichiometry and operon evolution, and propose a new cis-element-based framework to model the direction and rate of SRPS operon evolution.

\section{Acknowledgements}

This work was supported by University of Chinese Academy of Sciences Scholarship for International PhD students.

\section{Author contribution}

YB and JX designed the study; YB performed the computational analysis; YB and JX analyzed the data; MHB and CX provided critical suggestions; YB and JX wrote the paper.

\section{Competing interests}

The authors declare no conflicts of interest.

\section{Data availability}


bioRxiv preprint doi: https://doi.org/10.1101/2021.06.12.447814; this version posted June 13, 2021. The copyright holder for this preprint (which was not certified by peer review) is the author/funder, who has granted bioRxiv a license to display the preprint in perpetuity. It is made available under aCC-BY-NC-ND 4.0 International license.

383 The data underlying this article are available in the article and in its online 384 supplementary material. 


\section{$9 \quad$ References}

386 Altschul SF, Gish W, Miller W, Myers EW \& Lipman DJ (1990) Basic local alignment 387 search tool. J Mol Biol 215: 403-410.

388 Angelini LG, Tavarini S \& Foschi L (2013) Spanish broom (Spartium junceum L.) as new 389 fiber for biocomposites: the effect of crop age and microbial retting on fiber quality. Vol. 3902013 p.`pp. Hindawi Publishing Corporation.

Bhaskar Y, Su X, Xu C \& Xu J (2021) Predicting Selective RNA Processing and Stabilization Operons in Clostridium spp. Front Microbiol 12: 1281.

Busch A, Kunert G, Heckel DG \& Pauchet Y (2017) Evolution and functional characterization of CAZymes belonging to subfamily 10 of glycoside hydrolase family 5 (GH5_10) in two species of phytophagous beetles. PloS one 12: e 0184305.

Fani R, Brilli M \& Lio P (2005) The origin and evolution of operons: the piecewise building of the proteobacterial histidine operon. J Mol Evol 60: 378-390.

Francino MP (2012) The ecology of bacterial genes and the survival of the new. Int J Evol Biol 2012.

Gogarten JP, Doolittle WF \& Lawrence JG (2002) Prokaryotic evolution in light of gene transfer. Mol Biol Evol 19: 2226-2238.

Hofacker IL (2003) Vienna RNA secondary structure server. Nucleic Acids Res 31: 34294093431.

410 Kimura M (1968) Evolutionary rate at the molecular level. Nature 217: 624-626.

411 Klug G (1993) The role of mRNA degradation in the regulated expression of bacterial 412 photosynthesis genes. Mol Microbiol 9: 1-7.

413 Kumar S, Stecher G \& Tamura K (2016) MEGA7: Molecular Evolutionary Genetics

414 Analysis version 7.0 for bigger datasets. Mol Biol Evol 33: 1870-1874. 
415 Lawrence JG \& Roth JR (1996) Selfish operons: horizontal transfer may drive the evolution

416 of gene clusters. Genetics 143: 1843-1860.

417 Ludwig H, Homuth G, Schmalisch M, Dyka FM, Hecker M \& Stülke J (2001) Transcription 418 of glycolytic genes and operons in Bacillus subtilis: evidence for the presence of multiple 419 levels of control of the gapA operon. Mol Microbiol 41: 409-422.

420 Macke TJ, Ecker DJ, Gutell RR, Gautheret D, Case DA \& Sampath R (2001) RNAMotif, an 421 RNA secondary structure definition and search algorithm. Nucleic Acids Res 29: 4724-4735.

McDonald AG, Boyce S \& Tipton KF (2008) ExplorEnz: the primary source of the IUBMB enzyme list. Nucleic Acids Res 37: D593-D597.

Nei M (2005) Selectionism and neutralism in molecular evolution. Mol Biol Evol 22: 23182342 .

Neupane P, Bhuju S, Thapa N \& Bhattarai HK (2019) ATP Synthase: Structure, Function and Inhibition. Vol. 10 p.^pp. 1-10.

Newbury SF, Smith NH \& Higgins CF (1987) Differential mRNA stability controls relative gene expression within a polycistronic operon. Cell 51: 1131-1143.

Osbourn AE \& Field B (2009) Operons. Cell Mol Life Sci 66: 3755-3775.

Pagès S, Valette O, Abdou L, Bélaïch A \& Bélaïch J-P (2003) A rhamnogalacturonan lyase in the Clostridium cellulolyticum cellulosome. J Bacteriol 185: 4727-4733.

Pinheiro BA, Proctor MR, Martinez-Fleites C, Prates JA, Money VA, Davies GJ, Bayer EA, FontesM CM, Fierobe H-P \& Gilbert HJ (2008) The Clostridium cellulolyticum dockerin displays a dual binding mode for its cohesin partner. J Biol Chem 283: 18422-18430.

Price MN, Arkin AP \& Alm EJ (2006) The life-cycle of operons. PLoS Genet 2: e96.

438 Price MN, Huang KH, Arkin AP \& Alm EJ (2005) Operon formation is driven by co439 regulation and not by horizontal gene transfer. Genome Res 15: 809-819.

440 Rochat T, Bouloc P \& Repoila F (2013) Gene expression control by selective RNA 441 processing and stabilization in bacteria. FEMS Microbiol Lett 344: 104-113. (cellulosome) of Clostridium acetobutylicum. FEMS Microbiol Lett 217: 15-22. 
444 Smith C, Heyne S, Richter AS, Will S \& Backofen R (2010) Freiburg RNA Tools: a web 445 server integrating INTARNA, EXPARNA and LOCARNA. Nucleic Acids Res 38: W373446 W377.

447 Suzuki R \& Shimodaira H (2006) Pvclust: an R package for assessing the uncertainty in 448 hierarchical clustering. Bioinformatics 22: 1540-1542.

449 Will S, Joshi T, Hofacker IL, Stadler PF \& Backofen R (2012) LocARNA-P: accurate 450 boundary prediction and improved detection of structural RNAs. RNA 18: 900-914.

451 Xu C, Huang R, Teng L, Jing X, Hu J, Cui G, Wang Y, Cui Q \& Xu J (2015) Cellulosome 452 stoichiometry in Clostridium cellulolyticum is regulated by selective RNA processing and 453 stabilization. Nat Commun 6: 6900.

454 Yang Z (2007) PAML 4: phylogenetic analysis by maximum likelihood. Mol Biol Evol 24: $455 \quad 1586-1591$. 
Figure 1. Composite SLs (CoSLs) in the cellulosomal operons from 13 Clostridial species. (A) Schematic representation of the SRPS operon via $\Delta \mathrm{G}$ of the harbored composite SLs. Upstream Controlled Unit (UCU) represents the region (which is upstream to a SL and can include multiple genes) that is regulated by a SL via the SRPS mechanism. (B) $\Delta \mathrm{G}$ of the harbored SLs in the cellulosomal operons from 13 Clostridium spp., showing skewness of the $\Delta \mathrm{G}$ within an operon and divergence of pattern among orthologous operons. (C) Composite SLs (CoSLs)-based tree of the cellulosomal operons using the orthologous SLs. Genes are colored based on the encoded protein.

\section{Figure 2. Structural alignment of SLs harbored in the cellulosome operons from 13} Clostridial species reveal the relationship between SLs and their associated genes. The multiple sequence alignment is shown for the five orthologous SLs: SL-1 (A), SL-2 (B), SL-3 (C), SL-5 (D) and SL-7 (E). SLs were aligned via sequence or structure using LocARNA and shown with the consensus structure in the Dot bracket form (middle). Compatible base pairs are colored based on the standard format, where the hue shows sequence conservation among the number of different types of compatible base pairs (C-G, G-C, A-U, U-A, G-U or U-G) in the corresponding columns. Color saturation decreases with the number of incompatible base pairs. The bar plot represents conservation of compatible base pairs (higher bar for higher conservation, and vice versa).

Figure 3. Proposing CoSLOE to quantitatively model the evolution of SRPS operons. In CoSLOE (Composite Stem-Loop based Operon Evolution), operon ER is calculated based on number of genes, number of SLs and coefficient of the variation (CV) of the $\Delta \mathrm{G}$-based (i.e., $\Delta \mathrm{G}$ of the CoSLs) ratio. $\Delta \mathrm{GSD}$ and $\overline{\Delta \mathrm{G}}$ represent the standard deviation and the mean, respectively. The ratio determines the selection pressure between two operons, i.e., ratio of 1 represents the neutral selection, while positive (or negative) selection occurs when ratio is $>1$ (or $<1$ ). These ratios in a clade of a tree determine the direction of the evolution, i.e. positive selection for species with ratio $>1$ and negative/purifying selection for species with ratio $<1$.

Figure 4. Evolution of the cellulosome-encoding SRPS operons based on CoSLOE. The two clades in the tree represent two different operon evolution scenarios yet with an identical evolutionary directional flow, i.e., the movement from root to leaf nodes defines purifying (i.e., negative) selection and the reverse movement (i.e., leaf to root) depicts diversifying selection (i.e., positive selection). Upstream Controlled Unit (UCU) represents the region (which is upstream to a SL and can include multiple genes) that is regulated by a SL via the SRPS mechanism. (A) In Clade 1 (with four species), positive selection resulted in distinct $\Delta \mathrm{G}$ of SLs (each corresponding to a UCU) in the outermost operon of the clade, while those operons with similar $\Delta \mathrm{G}$ are conserved in the leaf nodes. Variation in a UCU can be caused by gain/loss of the SLs along with the corresponding genes (i.e., depicting appearance and disappearance of new genes). (B) In Clade 2 (with three species), positive selection also led to distinct $\Delta \mathrm{G}$ of SLs (each corresponding to a $\mathrm{UCU}$ ) in the outermost species, suggesting identical evolutionary flow in both clades. However, the positively selected operons carry characteristics distinct from the leaf-node operons, despite an identical number of SLs and 
genes. Thus the change in the $\Delta \mathrm{G}$ of SLs can lead to operons with discrete function. Color gradient of genes represents positive (darker color) or purifying (light color) selection.

\section{Figure 5. Evolution of the ATP-synthase-encoding SRPS operons in the 13 Clostridial} species based on the CoSLOE model. (A) ATP synthase operons sequences were mapped sequence-wise according to the CoSL-based phylogeny, where the sequence similarity is shown by dark black (100\% similarity) and light black (64\% similarity) color gradient and the genes are colored based on their encoding protein. The ERs determine the positive and negative selection pattern (shown via green and red arrows, respectively). (B) The multiple sequence alignment of SL-3 from the ATP synthase operons in the 13 Clostridial species.

Figure 6. The genome-wide CoSLOE model defines the direction of organismal selection. (A) Genome-wide evolution of SRPS operons based on the CoSLOE model (Table S4). Orthologous SRPS operons are scattered across the genomes of C. cellulolyticum (Ccel), $C$. josui (Cjos), C. sp. BNL1100 (Cbnl), and C. papyrosolvens (Cpap), represented here in the form of chromosome ( $\mathrm{Cbnl}$ and $\mathrm{Ccel}$ ) and contigs (Cpap-31 and Cjos-2). Totally, five out of 25 SRPS operons follow the Ccel-Cjos-Cbnl-Cpap direction and the other $80 \%$ SRPS operons show a positive selection flow in the Cpap-Cbnl-Cjos-Ccel direction, i.e., from the leaf nodes to the root nodes. Dark and grey region/band represents operon (Op), and their thickness shows the length. Direction of arrows represents direction of selection pressure, either positive (red) or negative (black). (B) Link and distinction between CoSLOE and the existing models for operon evolution. Information derived from the CoSLOE model is highlighted in red. 


\section{$524 \quad 11 \quad$ Table Legends}

525 Table 1. Evolutionary ratio (ER) matrix for the cellulosome complex operons from the

52613 Clostridial species. (A) ERs for Clade-1 of CoSL-tree, where the subclades of Cpap-

527 Cbnl-Cjos-Ccel, Ccell-Cter-Chun and Cros-Cfel-Cace are colored with orange, yellow and 528 green respectively. (B) ERs for Clade-2 of CoSL-tree.

$529 \quad(\mathbf{A})$

\begin{tabular}{lcccccccccc}
\hline & Cpap & Cbnl & Cjos & Ccel & Ccell & Cter & Chun & Cros & Cfel & Cace \\
\hline Cpap & 1 & 0.99 & 1.75 & 0.91 & 2.50 & 2.28 & 1.37 & 10.21 & 9.72 & 1.32 \\
\hline Cbnl & 1.01 & 1 & 1.76 & 0.91 & 2.52 & 2.30 & 1.38 & 10.29 & 9.79 & 1.33 \\
\hline Cjos & 0.57 & 0.57 & 1 & 0.52 & 1.43 & 1.31 & 0.78 & 5.84 & 5.56 & 0.75 \\
\hline Ccel & 1.10 & 1.10 & 1.93 & 1 & 2.77 & 2.52 & 1.51 & 11.27 & 10.73 & 1.45 \\
\hline Ccell & 0.40 & 0.40 & 0.70 & 0.36 & 1 & 0.91 & 0.55 & 4.08 & 3.88 & 0.53 \\
\hline Cter & 0.44 & 0.43 & 0.77 & 0.40 & 1.10 & 1 & 0.60 & 4.47 & 4.26 & 0.58 \\
\hline Chun & 0.73 & 0.73 & 1.28 & 0.66 & 1.83 & 1.67 & 1 & 7.46 & 7.10 & 0.96 \\
\hline Cros & 0.10 & 0.10 & 0.17 & 0.09 & 0.25 & 0.22 & 0.13 & 1 & 0.95 & 0.13 \\
\hline Cfel & 0.10 & 0.10 & 0.18 & 0.09 & 0.26 & 0.23 & 0.14 & 1.05 & 1 & 0.14 \\
\hline Cace & 0.76 & 0.75 & 1.33 & 0.69 & 1.90 & 1.73 & 1.04 & 7.76 & 7.39 & 1 \\
\hline
\end{tabular}

(B)

\begin{tabular}{lccc}
\hline & Cloc & Csac & Cpun \\
\hline Cloc & 1 & 2.12 & 1.38 \\
\hline Csac & 0.47 & 1 & 0.65 \\
\hline Cpun & 0.72 & 1.54 & 1 \\
\hline
\end{tabular}


533 Table S1. Free energy $(\Delta G)$ of harbored SLs in the SRPS operons that encode cellulosome from 53413 Clostridial species.

Table S2. Ka/Ks values for the first gene in the cellulosomal operon from Cpap, Cbnl, Cjos and Ccel.

Table S3. The evolutionary ratio (ER) matrix for the ATP synthase operons from the 13 540 Clostridial species.

Table S4. The number of genes and SLs in all SRPS operons in the Ccel, Cjos, Cbnl and Cpap genomes.

Figure S1. Phylogenetic tree of 13 Clostrdial species based on the predicted $\Delta G$ of the SLs (CoSL-tree) (A) or the 16S rRNA sequences (16S-tree) (B).

Figure S2. Clade-wise representation of the SL-1 (A), SL-2 (B), SL-3 (C), SL-4 (D), SL-5 (E), 550 SL-6 (F), SL-7 (G), SL-3A (H), SL-2A (I) and SL-7A (J), based on their sequence and structure similarity.

Figure S3. Linear comparison of the nucleic acid sequences of the cellulosome operons from the 13 Clostridial species. 
Figure 1

(A)

Ratio prediction mechanism in SRPS operons

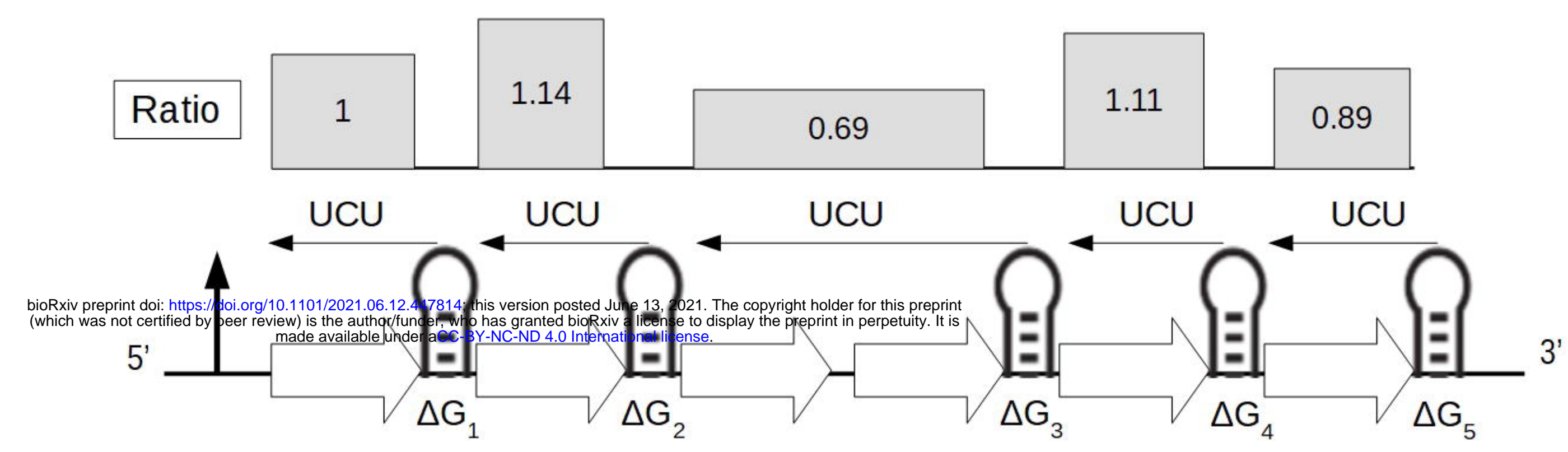

*UCU: Upstream controlled unit

(B)

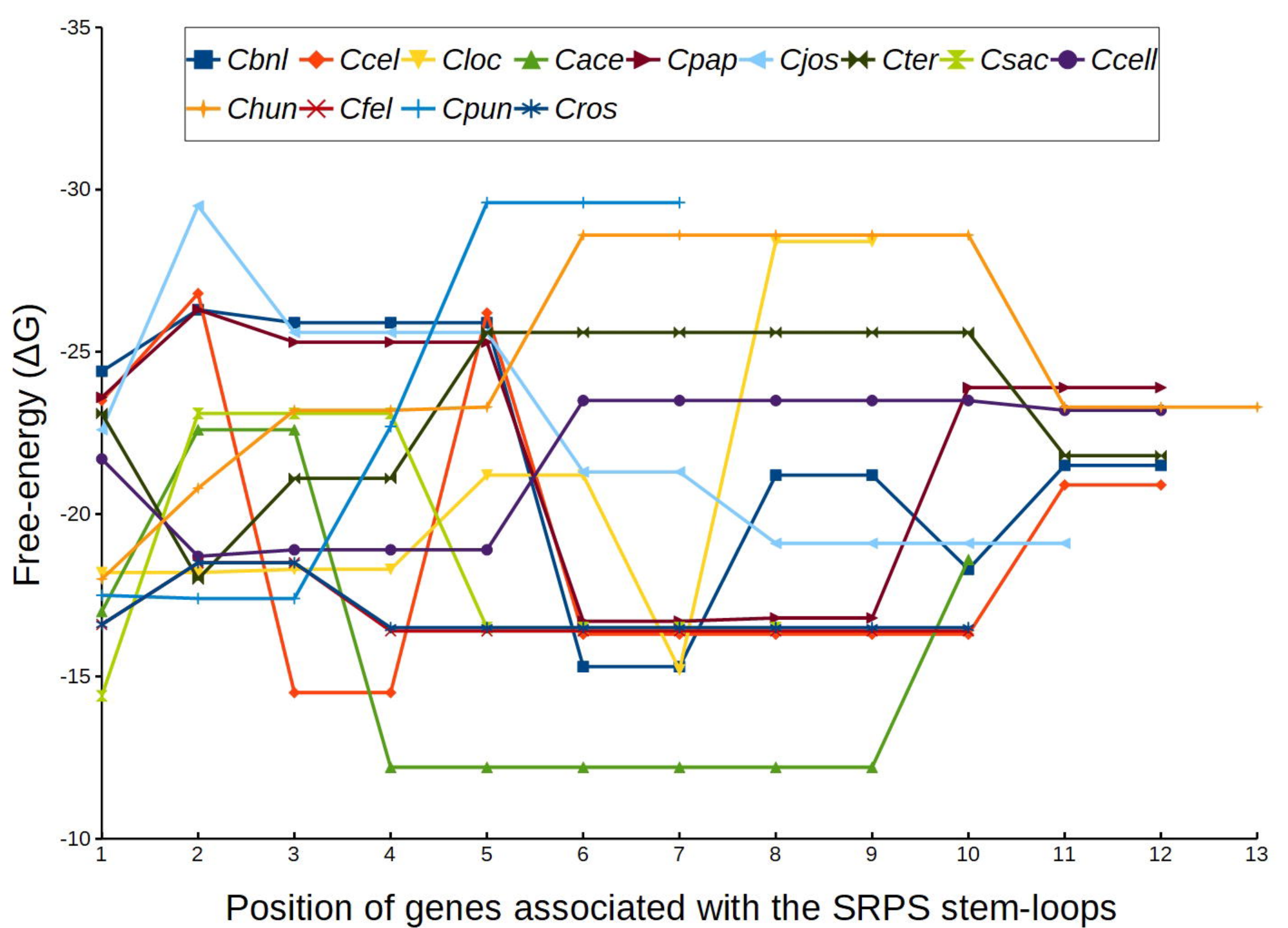

(C)

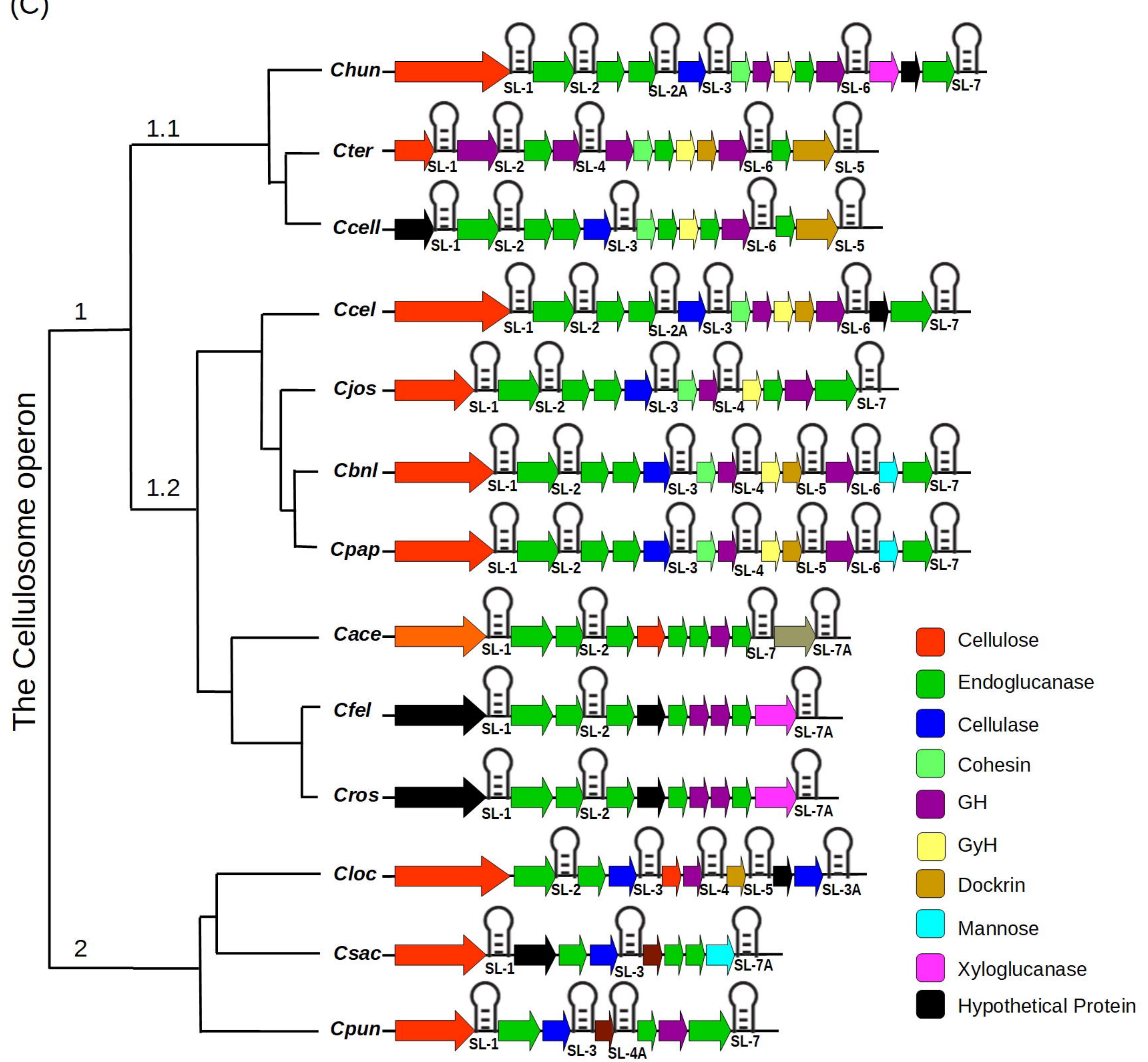


Figure 2

(A)

Stem-loop 1 (Cellulosomal operon)

$(((((((\ldots \ldots)(((((() \ldots \ldots \ldots \ldots \ldots \ldots \ldots \ldots \ldots \ldots \ldots \ldots)))))))))))))))$

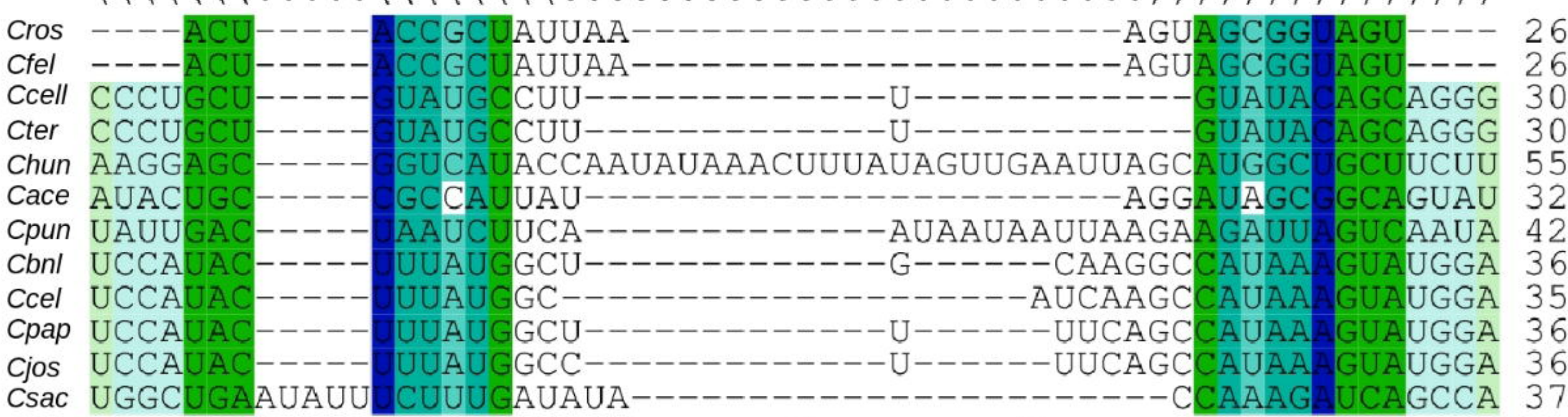

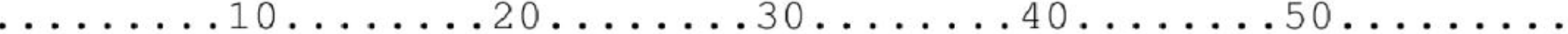

(B)

Stem-loop 2 (Cellulosomal operon)

$((((((((()(\ldots(((\ldots \ldots(((\ldots \ldots \ldots)))) \ldots \ldots))))))))))))))$

Cros GAUG UACCACU-UCAAAGUAUUA-UAAAGAUA--UAAAUGGAGAAGUGGU UAUC

Cfel GAUG ACCACU-UCAAAGUAUUA-UAAAGAUA--UAAAUGGAGAAGUGGU UAUC

ccell UAAG CCGGACAUGUAUG-UGAAU-U----UGAUUUCCUGUACUAUCCGGGCUUA

Cter UAAG CCGGACAUGUAUG-UGAAG-U----UGAUUUCCUGUACUAUCCGG CUUA

Cloc GGCU GACAAU-AU CAAGU - - - - - - - - - - - - - - - - - - - - - AUAUUGUC AGCU

Cace GGAU UACCAC-UUCAAAAUUUU-AUAGGAAUUUGAAUUGAGAAGUGGUA AUCU

Cbnl AGUC GGACGG-UAUUUGAUGUGAUUAAAUUAUUCAUUCUUGUACUGUCC GGCU

срар AGUC GGACGG-UAUUUGAUGUGAUUAACUAUUUCAUUCUUGUACUGUCC GGCU

ccel AGUC GGACAG-UAUUUGGUUUGAUUAAAUUACUCAUUCUUGUACUGUCC GGCU

Cjos AGUC GGACGG-UAUUUGAUGUGAUUAGAUUAUUCAUUCUUGUGCCGUCC GGCU
Chun AGUC GGACAUGUAUAUG-CUGAAGU----UUAUUCC---UGUACUGUCC GGCU

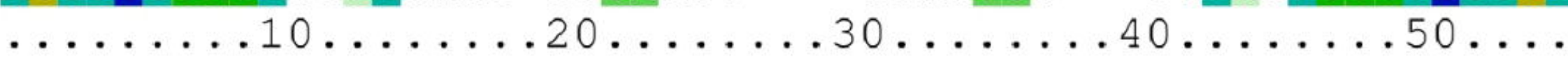

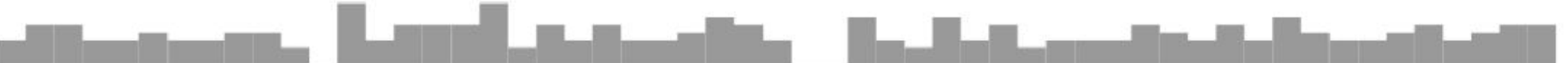

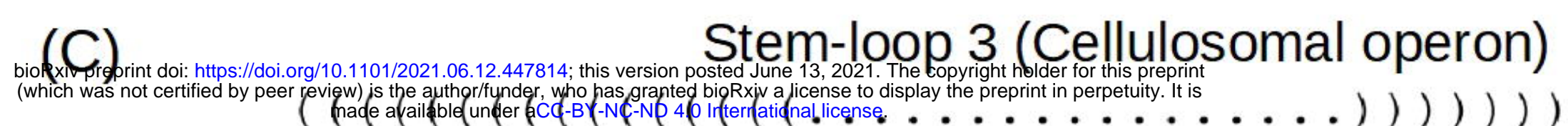

\begin{tabular}{|c|c|c|c|}
\hline $\mathrm{Cloc}$ & JAGAAUAAAAA & UGUAAAAAGG--UGAACUUUCUACA & UUUUUUAAUUUUAA \\
\hline Csac & GAAAAAAUUCC & UUGACAUAUUAAUUAUUUAUGUCAA & GGGAUUUUUUUUU \\
\hline Cbnl & JGAACAAUAGG & AUGC------------UUUAUGCAU & CCUAUUGUUCC \\
\hline Cjos & GAACAAUAGG & AUGCUU----------UUUAUGCAU & AUUG \\
\hline Ccel & & $--C U A G U G C A U$ & 200 \\
\hline Срар & AACAAUAGG & AUGCA------------UAAAGCAU & CCUAUUGUUC \\
\hline Chun & GAACUAUAGA & GGUCAG --------- CUAAUGAUC & UCUAUAGUUC \\
\hline Ccell & GAACUAUAGAA & UUUC---------UUGUGUAUUUGGG & UUCUAUAGUUC \\
\hline Cpun & UUAGUAUGGAU & AUAUAGAAU------AAAAAAAUAU & GUCCAUACUAL \\
\hline
\end{tabular}

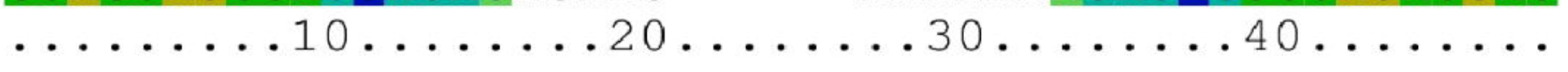

\section{Stem-loop 5 (Cellulosomal operon)}

$(((((((((()(\ldots((((\ldots \ldots \ldots \ldots \ldots)))))))))))))))))$ ccell AGUGGUAUGAGCGUCUUUUGAACAAUUAACCAAAGGGCUCGUACCACU cter AgUGGUAUGA GGCCUUUUGAACAAUUAACCAAAGGGCUCGUAUCACU cloc UCCUACAAGUGU-UAUAAAG----ACUUUCC--AUAACACUCGUAGGA Cbnl CUGUCCGGUAGA-GGA--------- - - - C--AUUUUUG CCGGACAG cpap CUGUCCGGCA GAAAUAUCU---------48 48 41 31 31

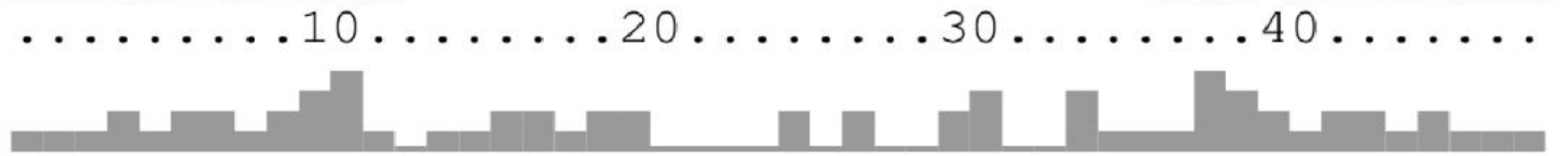

\section{Stem-loop 7 (Cellulosomal operon)}

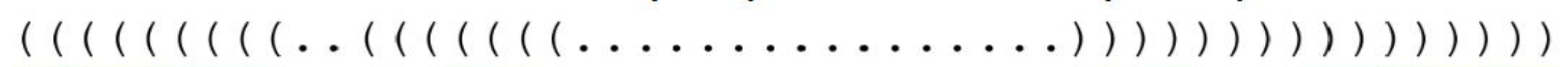
Ccel AUAAAAGAG---GCUGCCGAAA-------CUUCAGGCAGUCCUCUUUUAU 40 срар GAUAAA GUAAUG CUGCCGAAUA------UUUUCGGCAG CUAUUUUAUU 44 Cjos GAAAAAGAG--G GUGCCGUAAG--------UUUUCGUCA GCCUCUUUUUU 40 cpun GACGAAUAA--A ACUGUAGCAAAGAAAUUUGCUACAGUUUUUAUUCGUU 48 chun AGAGGGUGU--A GCCG--AA----------UUUACGGUAUACACCCUCU 36 Cbnl AAAAAUAGG--CU ACCGGAAA----------UUUCCGGC GCCUAUUUUU 38 Cace AUAACAGG-UGA GUUUUA---------------GACAUCUCUGUUAU 32

$\ldots \ldots 10 \ldots 20 \ldots 30 \ldots \ldots$ 


\section{Figure 3}

\section{Composite Stem-Loop based Operon Evolution (CoSLOE) model}
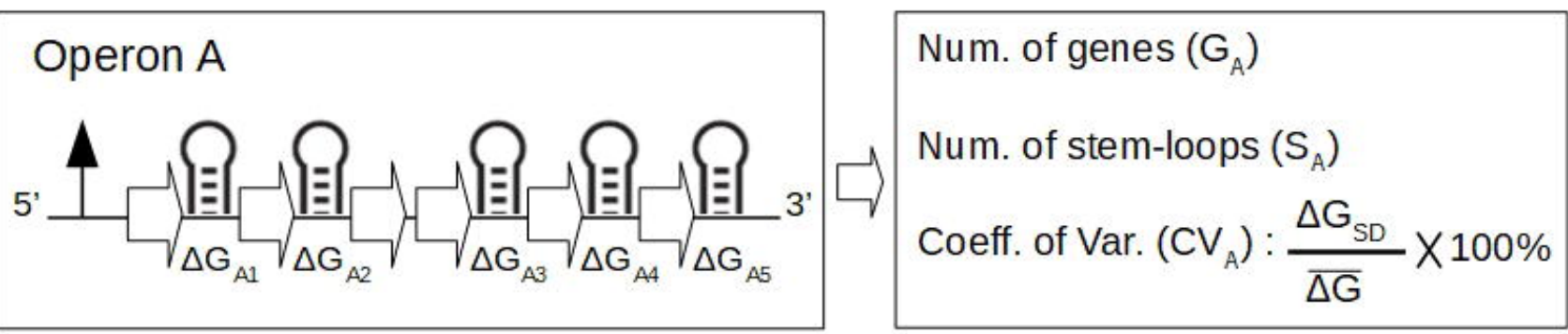

\section{Operon B}
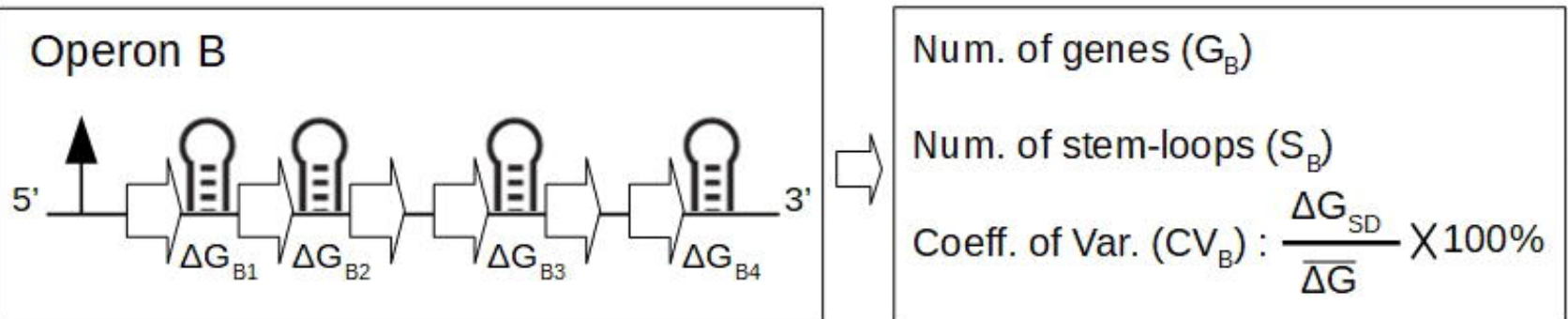

Operon evolution equation (for ideal condition)

$$
\frac{\mathrm{G}_{\mathrm{A}}}{\mathrm{G}_{\mathrm{B}}} \times \frac{\mathrm{S}_{\mathrm{A}}}{\mathrm{S}_{\mathrm{B}}} \times \frac{\mathrm{CV}_{\mathrm{A}}}{\mathrm{CV}_{\mathrm{B}}}=1
$$




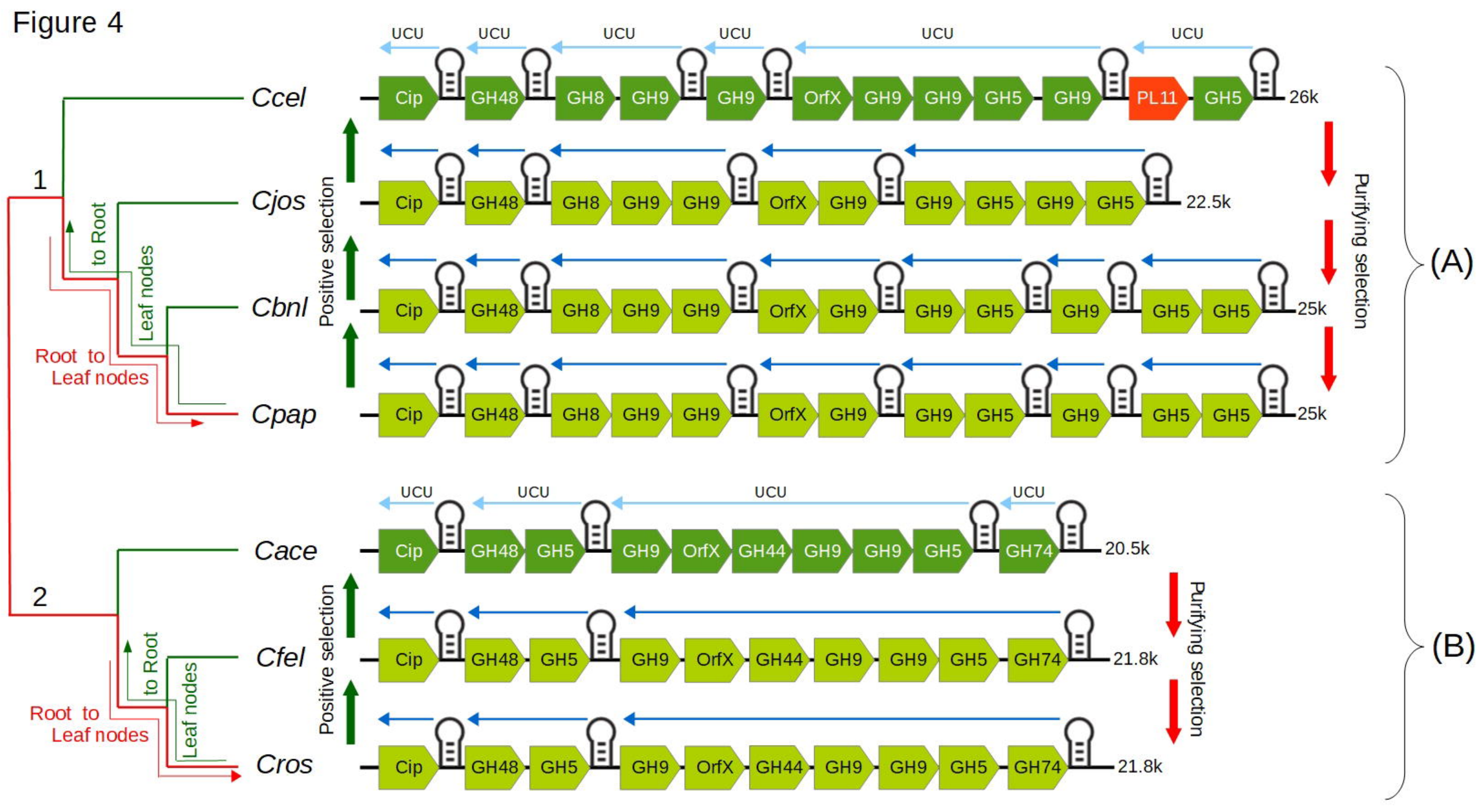




\section{Figure 5}
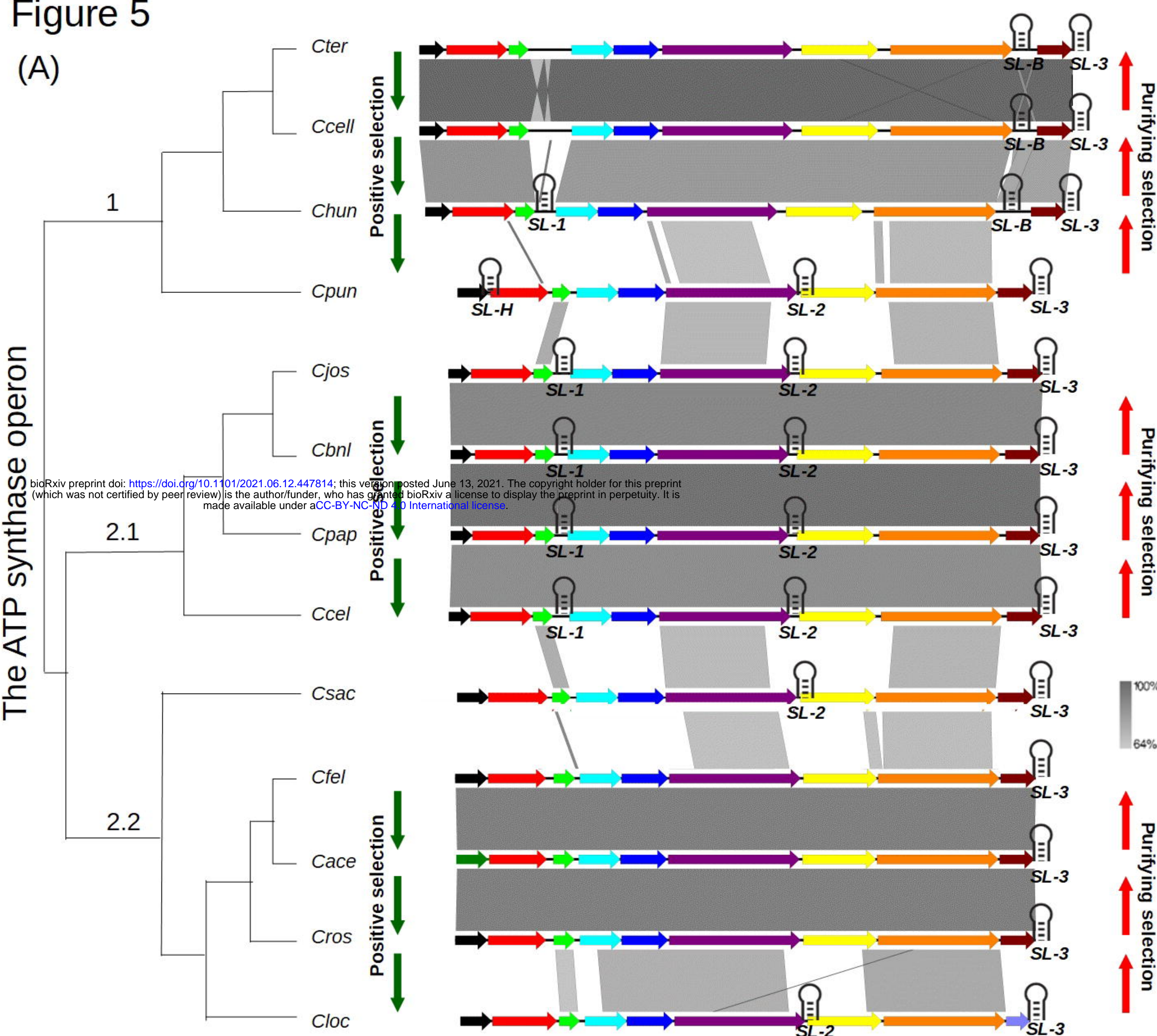

(B)

Stem-loop 3 (ATP synthase operon)

$(((((((((()(((((((\ldots \ldots \ldots \ldots)))))))))))))))))))$

cjos AGA

cbnl AGA GCCUAUACUGGAUUUUUA-------AAUAAAUCUUGUAUAGGCUCU 42

Chun AGACCCCAAAACACCCCGCCGGCGAAA--GGCGGGGUGUUUUGGGGUCU 47

ccell ACCCAAA A ACACCCCGCCUU- - - - - - UCGGCGGGGUGUUUUUGGGU 40

Cter ACCCAAAAACACCCCGUCUU-- - - - - - UCGGCGGGGUGUUUUUGGGU 40

cfel AAAAAACCGUGGAUUUUU - - - - - - - - - - - - - UUCGCGGUUUUUU 31

cace AAA AACCGUGGAAUAAA-- - - - - - - - - - - UCCACGGUUUUUU 31

cloc AAA AAgUGCUGAAUCCUAA-- - - - - - UUGGGAUUCAACACUUUUUU 40

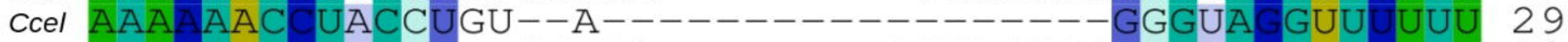

срар AAA GAAA GCUGUACACUAAGA-- - - - AAUUGGUGUACAGCUUUUUUU 42

cpun UAA ACGUACCUCUUUAUAUAGAAAAACUAUGUAAAGAGGUACGCUUUA 49

cros AAA $\mathrm{ACG}$ GGAUUUU---- $\mathrm{AU}$ -

Csac CUUUUAUAUAGGUGU-AA-- - - - - - - - - - - AUCUAUAUAAAAG 30 


\section{Figure $6(\mathrm{~A})$}

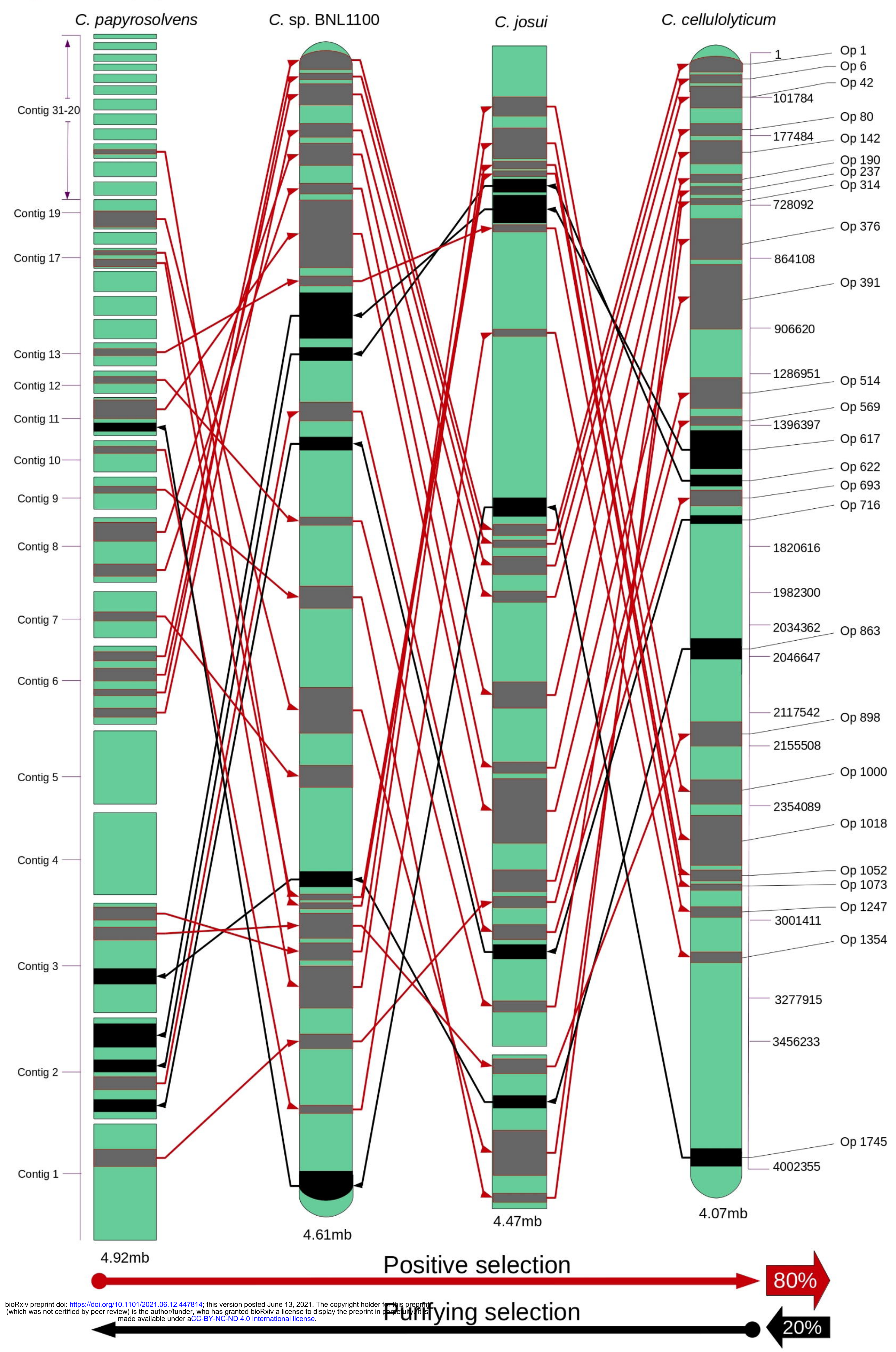

(B)

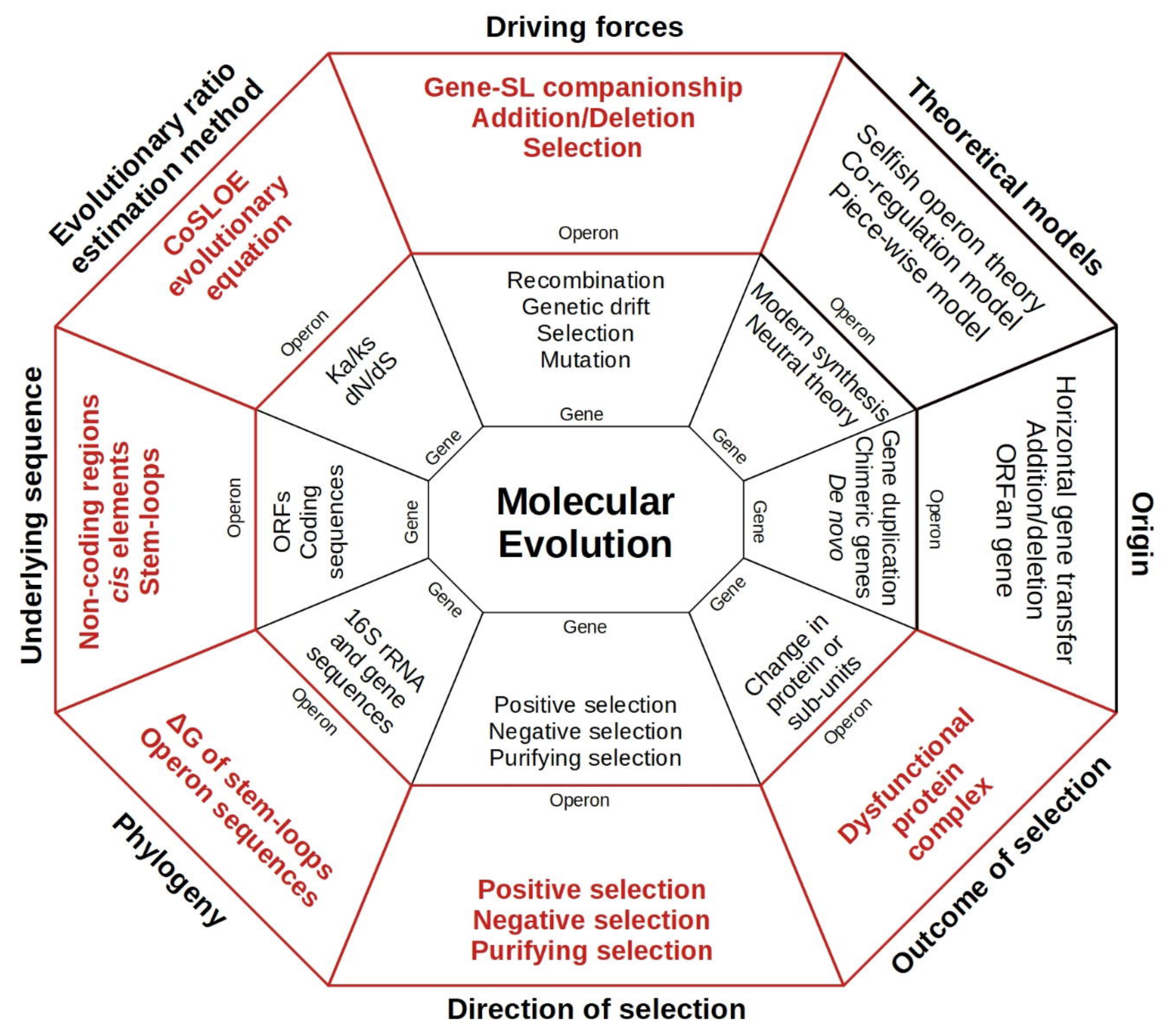

